\title{
Herbal Medicine for the Treatment of Vascular Dementia: An Overview of Scientific Evidence
}

\author{
Dennis Chang, ${ }^{1}$ Jianxun Liu, ${ }^{1,2}$ Kellie Bilinski, ${ }^{1}$ Li Xu, ${ }^{2}$ Genevieve Z. Steiner, ${ }^{1}$ \\ Sai W. Seto, ${ }^{1}$ and Alan Bensoussan ${ }^{1}$ \\ ${ }^{1}$ National Institute of Complementary Medicine, Western Sydney University, Penrith, NSW 2751, Australia \\ ${ }^{2}$ Xiyuan Hospital, China Academy of Chinese Medical Sciences, Chaoyang District, Beijing, China \\ Correspondence should be addressed to Dennis Chang; d.chang@westernsydney.edu.au
}

Received 2 September 2016; Accepted 31 October 2016

Academic Editor: Mark Moss

Copyright (C) 2016 Dennis Chang et al. This is an open access article distributed under the Creative Commons Attribution License, which permits unrestricted use, distribution, and reproduction in any medium, provided the original work is properly cited.

\begin{abstract}
Dementia is a leading cause of mental and physical disability. Vascular dementia (VaD) is the second most common cause of dementia after Alzheimer's disease (AD) constituting 10-15\% of the dementia population. Currently there are no approved pharmaceutical options for $\mathrm{VaD}$ and the conventional anti-AD therapies provide only modest, short-term relief of symptoms associated with VaD. Herbal medicines have been used for the management of dementia-like symptoms for centuries and may provide viable therapies for $\mathrm{VaD}$ due to their multicomponent and multitarget approach. This review is designed to provide an updated overview on the current status of herbal medicine research, with an emphasis on Chinese herbal medicine, for the treatment of $\mathrm{VaD}$ or dementia. A case study is also provided to demonstrate the development process of a novel standardized complex herbal formulation for $\mathrm{VaD}$. The article reveals some preliminary evidence to support the use of single and complex herbal preparations for $\mathrm{VaD}$ and dementia. Multiple issues in relation to clinical and preclinical research have been identified and future research directions are discussed.
\end{abstract}

\section{Introduction}

Dementia is a syndrome associated with progressive impairments in memory and learning ability, cognitive skills, behaviour, activities of daily living, and quality of life. There are more than 47.5 million people with dementia worldwide and 7.7 million new cases are added to the dementia pool each year [1]. In Australia, there are over 353,800 people living with dementia and the number is estimated to increase to 900,000 by the year of 2050 [2]. Dementia has surpassed cerebrovascular disease and lung cancer to become the 2nd leading cause of death in Australia [3].

There are numerous types of dementia, among which vascular dementia $(\mathrm{VaD})$ is the second most common cause after Alzheimer's disease (AD). Other common forms of dementia include Parkinson's disease, dementia with Lewy bodies, frontotemporal dementia, Huntington's disease, and alcoholrelated dementia. $\mathrm{VaD}$ is associated with cerebrovascular and cardiovascular diseases and constitutes $10-15 \%$ of dementia cases in western countries. In developing countries, the prevalence of $\mathrm{VaD}$ is higher, accounting for around $30 \%$ of the dementia prevalence, which is partially due to poorer control of cardiovascular risk factors [4]. VaD often coexists with other forms of dementia especially AD. Indeed, postmortem studies reveal that over $40 \%$ of clinically diagnosed $\mathrm{VaD}$ cases also have $\mathrm{AD}$ type of neurodegenerative pathology, which is the most common type of mixed dementia [5].

Currently cholinesterase inhibitors and glutamate receptor antagonists are the most effective pharmaceutical options for the treatment of $\mathrm{AD} \mathrm{[6].} \mathrm{These} \mathrm{medications} \mathrm{have} \mathrm{also} \mathrm{been}$ used off-label in some countries for the symptomatic relief in people with $\mathrm{VaD}$, but the safety and the long-term therapeutic benefits of these interventions in $\mathrm{VaD}$ remain uncertain.

In the absence of satisfactory pharmacological therapies, many people with $\mathrm{VaD}$ or dementia and their carers turn to complementary medicine. The common complementary medicine interventions for $\mathrm{VaD}$ and dementia and dementia risk-reduction include herbal medicine, acupuncture, 


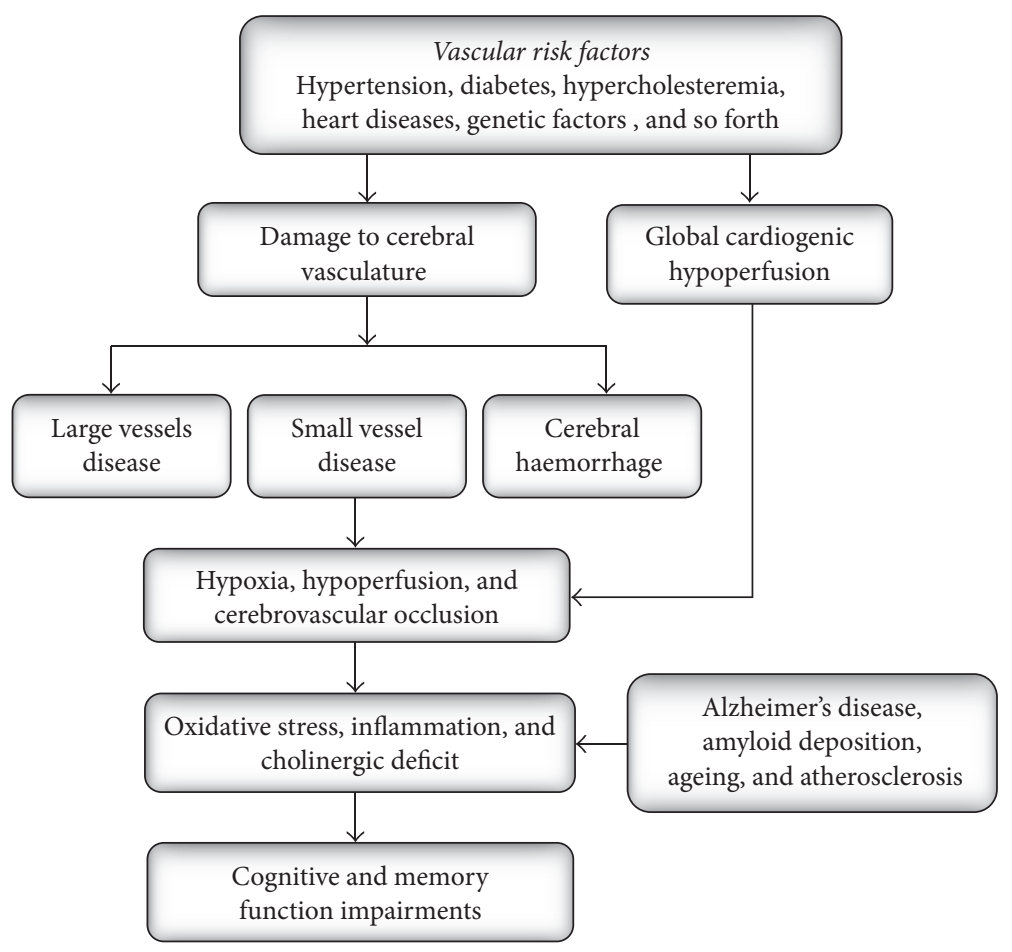

FIGURE 1: Pathophysiological mechanisms for vascular dementia.

nutraceuticals, yoga, tai chi, and music therapy. The use of herbal medicine for the treatment of ageing-related disorders was documented in the literature more than 2000 years ago in ancient China where herbal remedies were used to boost memory function and increase longevity [7]. Early preclinical and clinical evidence exists to support the use of herbal medicines either as single herbal preparations or as complex herbal formulations for $\mathrm{VaD}$. This review paper aims to provide an updated overview of evidence to support some of the commonly used herbs and herbal combinations with an emphasis on Chinese herbal medicine for the treatment of the disease. Issues and challenges associated with herbal medicines are discussed, and a case study is provided to demonstrate the development process of a novel complex herbal formulation for $\mathrm{VaD}$ that takes advantage of modern pharmaceutical and pharmacological technologies.

\section{Pathophysiology and Therapeutic Options for Vascular Dementia}

Cognitive impairment (especially executive dysfunction) is the primary symptom of $\mathrm{VaD}$, which can also cause a disturbance in mood and behaviour and reduce of quality of life. According to the blood vessels involved and the pathological processes, $\mathrm{VaD}$ can be divided into large vessel dementia (multiple infarcts or multi-infarct dementia), small vessel dementia (small vessel disease and microinfarction), strategic infarct dementia, hypoperfusive dementia, dementia related to angiopathies (hypertension, amyloid), haemorrhagic dementia, and familial vascular dementia.
The main risk factors associated with $\mathrm{VaD}$ include hypertension, hyperlipidemia, diabetes, genetic disposition, cardiac diseases, physical inactivity, and obesity [8]. The pathophysiology of $\mathrm{VaD}$ is complex. It incorporates interactions between vascular aetiologies (cerebrovascular disorders and vascular factors), changes in the brain (infarcts, white matter lesions, and atrophy), and host factors (age, education) [9]. The final common aetiopathogenic pathway usually attributes to a hypoxic, hypoperfusive, or occlusive process resulting in ischemic damage in various areas of the brain with subsequent cognitive and memory function impairments (Figure 1) [10]. Other pathogenic factors such as AD, amyloid deposition, ageing, and atherosclerosis also contribute to $\mathrm{VaD}$ development via inflammation and oxidative stress [8].

Currently, effective pharmaceutical interventions for $\mathrm{VaD}$ are lacking. Standard treatment largely focuses on symptomatic management and prevention of additional brain damage via recognition and control of cardiovascular and cerebrovascular risks using, for example, antihypertensives, aspirin, statins, vascular care, antidiabetes, and lifestyle modification [11]. Several classes of anti-AD pharmaceutical agents are used off-label for symptomatic management in $\mathrm{VaD}$. Cholinesterase (ChE) inhibitors (donepezil, galantamine, and rivastigmine) and NMDA receptor antagonists (memantine) have shown some modest short-term clinical benefits in improving cognitive function; however, most of these studies fail to demonstrate significant improvements in global functioning, activities of daily living, and quality of life [12]. The majority of studies conducted so far are over a relatively short duration (5-6 months); therefore the long-term benefits 
and safety of these interventions in $\mathrm{VaD}$ have not been validated.

\section{Individual Herbs Used in VaD}

There is a long history of herbal medicine use to boost memory and cognitive functions and manage behavioral and psychological symptoms associated with dementia/VaD. Some of the most commonly used and studied herbs include Ginkgo biloba, Huperzia serrata, Curcuma longa, Panax ginseng, Panax notoginseng, Bacopa monnieri, Salvia miltiorrhiza, Crocus sativus, and Camellia sinensis. Table 1 summarises the nomenclature, key bioactive compounds, and mechanisms of action of these herbs.

3.1. Gingko biloba. Ginkgo biloba leaf extract (ginkgo) is one of the most studied medicinal herbs. Ginkgo leaf extract is widely used for ageing-related memory disorders in many European and Asian countries. The principal constituents of ginkgo include flavonol glycosides (e.g., quercetin and kaempferol) and terpenoids (e.g., ginkgolide and bilobalide) [13]. Preclinical studies suggest that ginkgo decreases oxygen radical discharge and proinflammatory functions of macrophages (antioxidant and anti-inflammatory), reduces corticosteroid production (antianxiety), and increases glucose uptake and utilisation and adenosine triphosphate (ATP) production [14]. Ginkgo also appears to improve blood flow through increasing red blood cell deformability and decreasing red cell aggregation, inducing nitric oxide production, and antagonising platelet activating factor receptors [14]. EGb761 (a standard ginkgo preparation) treatment was also shown to enhance proliferation of neural stem cells in subventricular zones and the dentate gyrus [15] and to accelerate the recovery of the pathological synaptic plasticity [16] in VaD models in rats. In an ageing rat model, EGb761 reduced circulating free cholesterol and brain $\beta$-amyloid precursor protein production [17].

In animal studies, the effects of ginkgo leaf extracts on neuroprotection and cognitive dysfunctions have been demonstrated in various cerebral ischemia models in rats $[18,19]$, mice $[20]$, and gerbils $[21,22]$. In a recent study in rats with two-vessel (bilateral common carotid arteries) occlusion-induced $\mathrm{VaD}$ model, treatment of bilobalide significantly improved the learning and memory ability of the animals in a Morris water maze task [23].

In healthy young adults, ginkgo treatment has been shown to improve speed of processing, working memory, executive function, and cognition [24]. In a study with 80 patients with vascular cognitive impairment (not diagnosed with dementia), a combined therapy of ginkgo extract with conventional treatment of aspirin over three months significantly improved executive function, attention, abstractthinking, delayed memory, and orientation when compared with the control group (aspirin treatment only) [25].

The evidence to support the use of gingko for dementia remains controversial. Although some clinical studies fail to show a significant difference between ginkgo and placebo in dementia groups [26], numerous clinical trials demonstrate that ginkgo improves memory loss and concentration and decreases anxiety in patients with dementia and/or $\mathrm{VaD}$. For example, a randomised, double blind, placebocontrolled trial of 216 participants with $\mathrm{AD}$ or vascular dementia showed a significant improvement in attention and memory function in the EGb761-treated group after 24week treatment [27]. In a more recent trial, 404 people with dementia (333 $\mathrm{AD}$ and/or mixed dementia and $71 \mathrm{VaD}$ ) were treated with $240 \mathrm{mg}$ EGb 761 or placebo over 24 weeks. The results demonstrate that gingko treatment significantly improves cognitive function and neuropsychiatric symptoms [28]. No difference was found between the $\mathrm{AD}$ and $\mathrm{VaD}$ groups. These effects have been confirmed by several metaanalyses, indicating that ginkgo treatment stabilises or slows decline in cognition, function, and behaviour $[26,29,30]$. A recently published systematic review, in which nine relatively high quality clinical trials (six studies included participants with $\mathrm{VaD}$ or mixed dementia) were recruited, reported that EGb761 not only enhances scores of neurocognition but also improves activities of daily living in patients with $\mathrm{AD}$ and/or $\mathrm{VaD} /$ mixed dementia [30].

3.2. Huperzia serrata. Huperzia serrata has a long history in Chinese medicine for use in conditions including strains, swellings, schizophrenia, myasthenia gravis, and organophosphate poisoning. The key bioactive components of Huperzia serrata belong to the lycopodium alkaloids family including huperzine A (HupA), huperzine B (HupB), hyperzinine, carinatumin $\mathrm{A}$, and carinatumin $\mathrm{B}$, all of which possess antiacetylcholinesterase properties [31]. In particular, HupA became known globally after the discovery in the 1980s for its use as a potent acetylcholinesterase (AChE) inhibitor in the treatment of dementia. In addition, HupA has also been shown to exert other pharmacological effects including antioxidant, anti-inflammatory, antiapoptosis, anti- $\beta$-amyloid peptide fragmentation, inhibition of oxygen-glucose deprivation, and NMDA receptor antagonism [32, 33] (Table 2).

The majority of clinical trials of HupA to date have been conducted in China and have investigated its effect in $\mathrm{AD}$ patients. A meta-analysis of HupA for the treatment of $\mathrm{AD}$ identified 11 studies (one open-label study, two case reports, and eight controlled clinical trials), among which 4 trials involving 474 patients (235 in the HupA treatment group and 239 in the control group) were included in the final analysis [34]. The results demonstrate that HupA (300-500 $\mu \mathrm{g} /$ day) significantly improves cognitive function, as assessed by the mini-mental state examination (MMSE) and activities of daily living (ADLs). Similarly, a recent Cochrane systematic review of six clinical trials with a total of 454 patients concluded that HupA treatment led to an improvement in general cognitive function, global clinical status, behavioral disturbance, and functional performance, with minimal side effects in AD patients [35]. Another systematic review and meta-analysis of 20 RCTs involving 1823 participants showed beneficial effects of HupA (200-800 $\mu \mathrm{g} /$ day) on improvement of cognitive function, as measured by MMSE, Hasegawa Dementia Scale (HDS), and ADL [36], although the authors note that findings should be interpreted with caution due to the poor methodological quality of the included trials. 


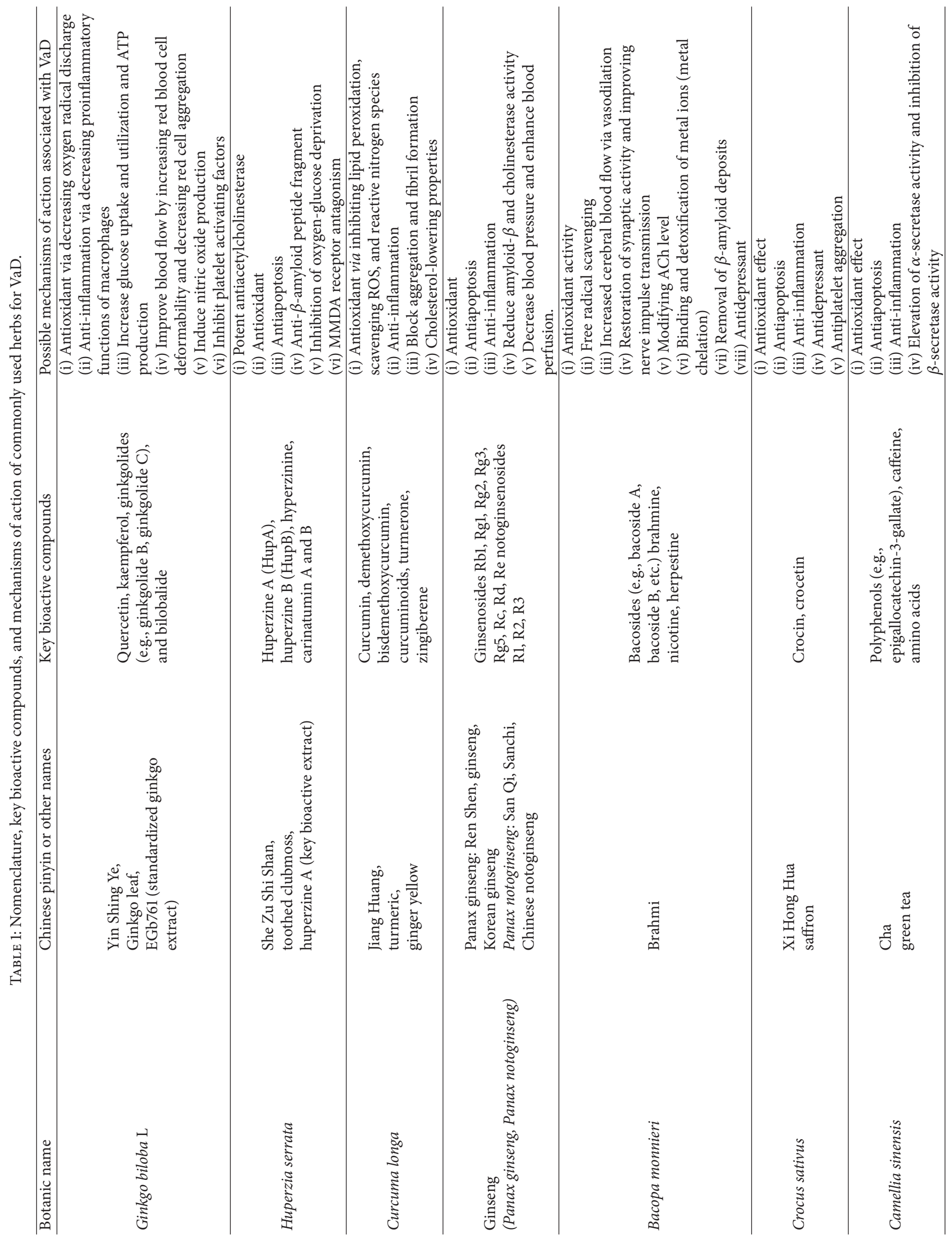


TABLE 2: Multitarget mechanisms underlying pharmacological effects of SLT components.

\begin{tabular}{|c|c|c|c|c|c|c|c|c|c|}
\hline \multirow{2}{*}{$\begin{array}{l}\text { Therapeutic targets } \\
\text { associated with } \mathrm{VaD}\end{array}$} & \multicolumn{3}{|c|}{ Ginseng } & \multicolumn{3}{|c|}{ Ginkgo } & \multicolumn{3}{|c|}{ Saffron } \\
\hline & Rg1 & $\operatorname{Re}$ & Rb1 & $\mathrm{Rd}$ & Ginkgo flavonoids & Ginkgolides & Total flavone-glycosides & Crocetin & Crocin \\
\hline Excitatory amino acid & & & $\mathrm{X}$ & $\mathrm{X}$ & $\mathrm{X}$ & $\mathrm{X}$ & $\mathrm{X}$ & & \\
\hline Energy depletion & & & & & & $\mathrm{X}$ & $\mathrm{X}$ & & \\
\hline Calcium overload & & & $\mathrm{X}$ & $\mathrm{X}$ & & $\mathrm{X}$ & $\mathrm{X}$ & & $\mathrm{X}$ \\
\hline Inflammation cascade & & & & $\mathrm{X}$ & & $\mathrm{X}$ & & & \\
\hline Oxidative stress & $\mathrm{X}$ & $\mathrm{X}$ & & & & $\mathrm{X}$ & $\mathrm{X}$ & & \\
\hline Cholinergic system & $\mathrm{X}$ & $\mathrm{X}$ & & & & & $\mathrm{X}$ & & \\
\hline Apoptosis & $\mathrm{X}$ & & $\mathrm{X}$ & & & & $\mathrm{X}$ & & \\
\hline Cytoskeleton & & & & & & $\mathrm{X}$ & $\mathrm{X}$ & & \\
\hline Antithrombus & $\mathrm{X}$ & & & & & & $\mathrm{X}$ & & \\
\hline Fibrinolysis & $\mathrm{X}$ & & & & & & $\mathrm{X}$ & & \\
\hline Platelet aggregation & & & & & $\mathrm{X}$ & $\mathrm{X}$ & $\mathrm{X}$ & $\mathrm{X}$ & $\mathrm{X}$ \\
\hline Cerebral circulation & $\mathrm{X}$ & & & & $\mathrm{X}$ & & $\mathrm{X}$ & & \\
\hline
\end{tabular}

Among studies which have included $\mathrm{VaD}$ patients, one Cochrane review investigated HupA for VaD but only identified one small study involving 14 participants in which HupA was found to be no better than placebo [37]. A subsequent meta-analysis of placebo-controlled RCTs of HupA on patients with $\mathrm{AD}$ and $\mathrm{VaD}$ identified eight $\mathrm{AD}$ and two $\mathrm{VaD}$ trials with 733 and 92 participants, respectively [38]. HupA treatment (100-500 $\mu \mathrm{g} /$ day) was shown to significantly improve the MMSE and ADL scores of $\mathrm{AD}$ and $\mathrm{VaD}$ patients, and longer treatment duration resulted in improved efficacy for $\mathrm{AD}$ patients. However, as noted in these reviews, the lack of quality data, small sample sizes of individual clinical trials, and short intervention periods limit firm conclusions about HupA's clinical efficacy, highlighting the need for rigorous randomised controlled trials with large sample sizes.

3.3. Curcuma longa. Curcuma longa (turmeric) is a food spice and colouring agent used in Chinese, Hindu, and Ayurvedic medicine for centuries has been applied in therapeutic preparations to treat numerous conditions such as pancreatitis, arthritis, cancer, and inflammatory, neurodegenerative, and digestive disorders. Curcumin and curcuminoids are the key bioactive components of turmeric consisting of three structurally closely related chemical components: curcumin, demethoxycurcumin, and bisdemethoxycurcumin [39]. Data from animal and/or in vitro studies suggests that curcumin can affect multiple pathological targets associated with dementia via inhibiting lipid peroxidation, scavenging reactive oxygen species (ROS), and reactive nitrogen species, inhibiting NF-kB activation, and its antiinflammatory actions $[31,40]$. It has also been suggested that curcumin is able to directly bind small beta-amyloid species to block aggregation and fibril formation [41].

Animal studies have shown that curcumin offers protective effects against $\mathrm{VaD}$ by exerting antioxidant and antiinflammatory effects. A lower prevalence of $\mathrm{AD}$ in some Asian populations has been attributed to a curcumin-rich diet. One population-based study of 1,010 Asian seniors without dementia showed that consumption of turmeric containing curry was associated with improved cognitive function as measured by MMSE [42]. Based on these findings several clinical trials have been initiated [43-45]. One pilot randomised, double blind, placebo-controlled trial evaluated the pharmacokinetics and effects of curcumin supplementation (1-4 g/day) over six months in $34 \mathrm{AD}$ patients [44]. The results of the study showed slight improvements in MMSE scores without significant side effects; however interpretation of these findings is limited due to the small sample size, short follow-up period, and lack of cognitive decline in the placebo group. In another 24 months, randomised controlled trial in 36 patients with mild-moderate $\mathrm{AD}$ and 2,000 mg and 4,000 mg/day of curcumin C3 Complex ${ }^{\circledR}$ over 24 weeks failed to demonstrate any clinical or biochemical evidence of efficacy of curcumin in AD [46]. The lack of positive findings may be somewhat attributed to curcumin's relatively low solubility and bioavailability. Further studies are required focusing on the active components of curcumin to determine the therapeutic value of curcumin in the treatment of dementia.

3.4. Ginseng. Panax ginseng (Ren Shen) and Panax notoginseng (San Qi) are two important members of the ginseng species and have been used for centuries in Chinese medicine to treat atherosclerosis, hypertension, thrombosis, external injury, and pain. In addition, ginseng has shown therapeutic benefits for learning and memory and may be useful in developing supplements for the prevention or potential treatment of $\mathrm{AD}[47,48]$. The principal bioactive components of ginseng are ginsenosides (e.g., ginsenosides Rg1, Rg3, and Rg5), which have been suggested to have antioxidant, antiinflammatory, and antiapoptotic effects [49]. In addition, ginsenoside $\operatorname{Rg} 5$ has been shown to reduce amyloid- $\beta$ and cholinesterase activity [50], while ginsenoside $\operatorname{Rg} 3$ has also been shown to promote $\beta$-amyloid peptide degradation via enhancing gene expression [51-54]. In addition, research 
demonstrates that Panax ginseng decreases blood pressure and improves blood circulation via vasodilation activities [55].

Ginseng is widely used to treat dementia-like symptoms in many Asian countries; however the majority of studies examining its effects on cognition have been studied in animals and healthy individuals. Clinical trial data suggests that ginseng modestly improves thinking and working memory in healthy volunteers. [56, 57]. Two open-label trials showed that 12 -week treatment with ginseng improved $\mathrm{AD}$ Assessment Scale-Cognitive Subscale (ADAS-cog) scores in AD participants $[58,59]$.

Two recent small open-label trials demonstrate the potential therapeutic benefits of Panax ginseng for $\mathrm{AD}[59,60]$. In the former study, which showed significant effects on ADAScog and Clinical Dementia Rating (CDR) following 24-week treatment of low or high dose ( $4.5 \mathrm{~g}$ or $9 \mathrm{~g} /$ day) Panax ginseng compared to controls [58], subjects were followed up for further 2 years during which time cognitive function was evaluated every 12 weeks using the ADAS and the Korean version of the MMSE (K-MMSE). In the long-term efficacy evaluation of the effect of Panax ginseng, cognitive function was sustained for the follow-up period. In the latter study, in which $87 \mathrm{AD}$ participants (58 in the ginseng group and 39 in the control group) were involved, 12-week treatment with Panax ginseng powder ( $4.5 \mathrm{~g} /$ day) produced significant improvements in ADAS-cog and MMSE scores [59]. Clinical benefits have also been demonstrated after Panax ginseng is combined with ginkgo in improving cognitive function in healthy subjects [14, 24, 61-63].

Less research has been conducted on Panax notoginseng. One randomised controlled trial in 40 people with $\mathrm{VaD}$, which compared the effect of 12-week supplementation with Panax notoginseng to duxil, a drug that increases oxygen in brain tissue, showed that memory function significantly improved in those given the herb [64]. In a trial with 64 older adults with lacunar infarction (cerebrovascular disease), the effects of the injectable form of Panax notoginseng extract (Xueshuantong) were investigated. Four-week treatment of Xueshuantong significantly increased relative cerebral blood flow and improved the ADL scores, although MMSE scores showed no marked changes [65]. Large scale, long-term studies using standardized extracts are required to confirm the clinical efficacy of ginseng therapy in dementia and $\mathrm{VaD}$.

3.5. Bacopa monnieri. Bacopa monnieri (Brahmi) has been traditionally used in Ayurvedic medicine to treat conditions including pain, asthma, fever, inflammation, and memory decline [66]. Various mechanisms may be involved in the neuroprotective and memory enhancing effects of Brahmi such as increasing antioxidant activity [67], free radical scavenging [68], binding and detoxification of metal ions [69], modifying levels of acetylcholine [68], and increasing cerebral blood flow via vasodilation [70]. The constituents responsible for improving learning and memory are attributed to steroidal saponins and bacosides A and B [71]. Bacosides enhance kinase activity and neuronal synthesis, which is linked with the restoration of synaptic activity, ultimately improving nerve impulse transmission [72].
Brahmi improves motor learning, acquisition, and retention and delays extinction of newly learned behaviour in animals [73]. A series of clinical studies have demonstrated the acute and chronic neurocognitive effects of Brahmi in healthy elderly populations [74]. A systematic review of six randomized controlled trials using a dose of 300-450 mg Brahmi per day showed that the compound preferentially enhances secondary memory, although the duration of supplementation in these trials was inadequate to substantiate the effects of Brahmi on cognition [75]. The Australian Research Council Longevity Intervention (ARCLI) study, a randomised, double-blind, placebo-controlled, 3arm parallel-group clinical trial is currently underway in attempt to overcome this issue by examining the effect of 12month administration of Brahmi on cognitive performance in 465 healthy participants [76]. A study to assess the effects of 6-month treatment of CDRI 08, a standardized Brahmi extract on cognitive function in $\mathrm{AD}$ patients, is also being planned by the same team [74].

3.6. Crocus sativus. Crocus sativus (Xi Hong Hua) commonly known as saffron is used in Chinese medicine as antidepressant, antispasmodic, and anticatarrhal. Data from in vivo and in vitro studies demonstrate that saffron possesses anti-inflammatory, antioxidant, and antiapoptotic properties [77]. Saffron extract has been shown to improve learning and memory function in ethanol-induced memory impairment in mice and to ameliorate cerebral ischaemia induced oxidative damage in the rat hippocampus [78, 79]. Crocin, the principal constituent of saffron and a strong antioxidant, has been suggested to be largely responsible for saffron's protective effect on the central nervous system [80]. It has also been suggested that saffron can act as an antidepressant and antiplatelet agent [81], both of which may offer additional benefits for people with dementia/VaD.

In more recent years, saffron has been used for neurological conditions [82]. A 22-week double-blind RCT in $\mathrm{AD}$ participants showed that saffron $(30 \mathrm{mg} / \mathrm{day})$ resulted in comparable improvements in cognition to donepezil (10 mg/day) although better tolerated [83]. Another 16-week double-blind trial in $\mathrm{AD}$ participants, comparing the same dose of saffron with placebo, showed saffron supplementation resulted in significantly better outcomes in cognitive function than placebo $[83,84]$. These findings support the need for larger trials over a longer duration.

3.7. Camellia sinensis. Camellia sinensis, commonly known as tea, is widely consumed as a health beverage, especially in the form of green tea in Asia. The chief bioactive components of tea are polyphenols, caffeine, and amino acids. Catechin polyphenol constituent, epigallocatechin-3-gallate (EGCG), is the most potent bioactive component of green tea. ECCG been shown to exert neuroprotective/neurorescue activities via a wide range of mechanisms including downregulation of proapoptotic genes, elevation of $\alpha$-secretase activity, and inhibition of $\beta$-secretase activity, anti-inflammation, scavenging of ROS, and stabilisation of mitochondrial function $[31,85,86]$. 
Animal and epidemiological studies have suggested that drinking green tea confers protection to the brain against the ageing process. An inverse correlation between tea consumption and the incidence of $\mathrm{AD}$ and other neurodegenerative diseases has been suggested [86], although longitudinal and cross-sectional studies investigating the effect of green tea on cognitive function have produced mixed findings.

A cross-sectional study assessing the effect of green tea consumption on cognitive function in 1,003 Japanese participants aged over 70 years showed that daily consumption of two or more cups of green tea $(100 \mathrm{~mL} / \mathrm{cup})$ was associated with a lower prevalence of cognitive impairment [87]. A recent double-blind, counterbalanced, within-subjects study which compared the effect of $27.5 \mathrm{~g}$ green tea extract on working memory in 12 healthy subjects showed that green tea increased working memory, suggesting that green tea may be effective for the treatment of cognitive impairments in disorders such as dementia [88]. Another prospective population-based study of 723 Japanese participants with normal cognitive function at baseline found that the incidence of dementia was significantly lower in those who consumed green tea 1-6 days/week compared to those who did not consume green tea [89]. In contrast, a double-blind randomised controlled study assessing the effects of green tea consumption ( $2 \mathrm{~g} /$ day) in 33 nursing home residents with cognitive dysfunction was unable to show a significant improvement in cognitive function as assessed by MMSE [90].

In summary, multiple herbs have demonstrated potential therapeutic benefits for improving cognitive function in dementia and/or $\mathrm{VaD}$. However, most of the evidence comes from preclinical research, and many of these findings have not been directly validated in people with $\mathrm{VaD}$. In addition, plasma concentrations of the bioactive components of these herbs are generally below the levels able to generate meaningful pharmacological activity. One possible explanation for these clinical effects is that these bioactive components interact synergistically leading to greater pharmacological/clinical outcomes than predicted by the activity of individual components. However, the evidence to support this theory is generally lacking and further research is needed to assess synergism of herbal medicine, as detailed below in Section 4.1.

\section{Complex Herbal Formulations}

4.1. Mechanisms and Synergistic Effects. Multiple herbs are often combined in complex formulations in some traditional medical systems for the treatment of various diseases. Theoretically, this multicomponent and multitarget approach may be ideal for diseases that have complex aetiologies and pathophysiologies such as $\mathrm{VaD}$.

In TCM, the use of multiherbal therapies in which up to 20 herbs are used underpins its unique philosophy and holistic approach. According to TCM theories, multiple herbs are included in a complex herbal formulation based on the principle of "Jun (emperor) - Chen (minister) - Zuo (assistant) - Shi (courier)." The "Jun" herb is the key therapeutic component of the formula directly targeting the disease, the "Chen" herb is included to relieve the accompanying symptoms of the disease and/or to enhance the effects of the key herb, the "Zuo" herb reduces toxicity of the herbal formula, and the "Shi" herb facilitates the delivery of active components of the formula to the target organs and/or harmonises their effects.

The Jun-Chen-Zuo-Shen theory describes a complex interactive relationship where the herbs in a complex herbal formulation interact synergistically to enhance distribution and/or ameliorate/prevent potential side effects. Some of these interactions are able to be explained pharmacologically. For example, bioactive components in herbal formulations interact improving their solubility and subsequent bioavailability, enabling them to affect multiple therapeutic targets associated with the disease and/or to enhance metabolism of toxic components thereby reducing side effects [91].

Evidence to support these beneficial interactions is very limited and results remain controversial. The paucity of data is partially caused by the lack of robust research methodologies to study the synergistic effects of multicomponent herbs or herbal formulations. The two methods most commonly used for studying synergism are the combination index (CI) and isobole method. Both methods have been developed to determine synergistic or antagonistic interactions between two or more single-entity agents acting on the same target/receptor and require the determination of a doseresponse relationship of the combination and its individual components [92]. However, these methods are inadequate for evaluation of synergy in complex herbal formulations where multicomponents interact with multiple therapeutic targets/receptors [93].

The use of the systematic analysis or system-to-system (S2S) method is gaining momentum in the study of multitarget synergistic actions. Taking advantage of computational sciences, the S2S approach integrates data from literature and experimental studies. S2S analysis is conducted via a docking process during which three-dimensional structures of individual compounds of interest are matched against the known structures of relevant key therapeutic protein targets associated with the disease using computer software. However, there is general lack of information on the chemical and pharmacologic properties of bioactive components of many herbal medicines and therefore the use of the S2S method in the study of complex herbal formulations remains a challenge [92].

\subsection{Clinical Evidence of Complex Herbal Formulations for} $\mathrm{VaD}$. The current clinical evidence to support the use of complex herbal formulations for dementia and/or $\mathrm{VaD}$ remains weak and controversial. There are only a limited number of reports published in English that examine the effectiveness of complex herbal formulations for VaD. For example, in a randomised, double-blind, placebo-controlled trial, the effects of a traditional Chinse herbal formulation, Bai Wei Di Huang Wan (consisting of 8 Chinese herbs), were examined in 33 patients with mild to severe dementia. Although the authors did not specify the types of the dementia, $91 \%$ of the patients recruited exhibited neuroimaging evidence of cerebrovascular disease and therefore it is likely 
that these patients had $\mathrm{VaD}$ or mixed dementia. The authors found that 8-week treatment of Bai Wei Di Huang Wang formula significantly improved cognitive function (measured by MMSE) and ADL (measured by Barthel Index) when compared to the placebo [94]. However, the trial was not fully powered and the invention period was relatively short. Further studies with larger sample sizes and longer duration are required to confirm these results.

The effects of a seven-herb Kampo/Chinese medicine formula, Yokukan-san (Yi-Gan San in TCM) on neurocognitive function and behavioral and psychological symptoms, were investigated in an open-label trial in 13 people with $\mathrm{VaD}$ according to the National Institute of Neurological Disorders and Stroke-Association Internationale pour la Recherche et l'Enseignement en Neurosciences (NINDS-AIREN) diagnostic criteria. Yokukan-san consists of Angelica acutiloba, Atractylodes lancea rhizome, Bupleurum radix, Poria sclerotium, Glycyrrhizae radix, Cnidium rhizome, and Uncarie hook. Although 4-week treatment of Yokukan-san did not significantly change the MMSE scores, there was a significant change in the overall NPI (neuropsychiatric inventory) score and mean subscores for agitation and disinhibition after the treatment, suggesting potential neuropsychiatric benefits of the formula [95].

A large number of trials were conducted in China to evaluate various complex Chinese herbal formulations for the treatment of $\mathrm{VaD}$. A systematic review published in 2012 detailed 47 randomised controlled clinical trials (all conducted in China) involving 3,725 people with $\mathrm{VaD}$ (using diverse diagnostic criteria) in an effort to assess the safety and efficacy of herbal medicines for $\mathrm{VaD}$ [96]. Out of 43 studies where herbal medicines were used as monotherapies, 37 reported that the herbal interventions exerted significantly greater effects than the conventional medicines (piracetam, aniracetam, hydergine, etc.) or placebos. All 4 studies in which herbal medicines were used in conjunction with conventional medicines reported better neurocognitive efficacy outcomes compared to the conventional medicines alone. However, significant methodological issues were identified in these studies including no sample size calculation, inconsistent diagnostic criteria used, differences in baseline characteristics, inappropriate randomisation, and diverse outcome measures used (some studies used instruments developed in-house). In addition, some 43 different herbal/complex herbal preparations were used in these studies. Each of these methodological shortcomings seriously impacts on the significance of these clinical findings.

A more recent meta-analysis conducted by Gong et al. [97] using strict inclusion criteria (e.g., exclusion of studies using single herbs or with short duration) included 24 randomised clinical trials comprising 2043 people with $\mathrm{VaD}$ (all conducted in China), although no analysis of the $\mathrm{VaD}$ diagnostic criteria used in these studies was provided. In a subgroup analyses, complex Chinese herbal interventions significantly enhanced cognitive function (judging by MMSE scores) when compared to piracetam (in 10 studies) or placebos (in 3 studies). No difference in MMSE scores was identified between the Chinese herbal medicines and hydergine in 17 of the studies. Yet, herbal medicine treatments produced a greater improvement in ADLs compared to piracetam treatment in 5 of the studies. Having said this, similar methodological problems to the previous systematic reviews were identified in these studies.

In summary, a number of complex herbal formulations have been trialled clinically but mainly in China. There are numerous methodological problems with most of these clinical trials. In addition, no studies reported the standardization of their herbal interventions used in the trials. Mechanistic studies to evaluate mechanisms of action and synergy of these formulations are also lacking.

\section{SLT, a Case Study on the Development of a Complex Herbal Formulation for VaD}

Recognising the lack of therapeutic options for the treatment of $\mathrm{VaD}$, a combined team from the China Academy of Chinese Medical Sciences and Western Sydney University (authors of this article have contributed to the project in various ways) has been working together to develop a standardized complex herbal formulation, SLT (Sailuotong, previously known as WNK) for the treatment of VaD. SLT represents a new generation of herbal medicine whereby the chemical and pharmacological profiles have been clearly defined. Over the last 10 years, a substantial body of evidence has accumulated in support of the use of SLT for VaD. Some of the key data have been summarised in this section by way of providing an example to demonstrate the developmental process of new complex herbal interventions for the treatment of serious diseases such as VaD.

5.1. SLT Development Process. SLT formula consists of specific dosages of standardized Ginkgo biloba (ginkgo), Panax ginseng (ginseng), and Crocus sativus (saffron) extracts, designed for the treatment of $\mathrm{VaD}$. The herbs were selected based on their traditional use and existing clinical and pharmacological evidence (as summarised in Sections 3.1, 3.4, and 3.6). A number of bioactive components have been identified in ginseng, ginkgo, and saffron that have a variety of pharmacological effects associated with $\mathrm{VaD}$. The effects of these key bioactive components of SLT formula are summarised in Table 2.

In the development of SLT, bioassay-guided fractionations were used to determine optimal organic solventbased extraction methods for each herb individually. The optimal dose ratio and dosage regimen were determined through a series of pharmacological studies using various animal models. These models include $\mathrm{VaD} /$ ischemia models (e.g., bilateral common carotid artery occlusion model in rats, MCAO-induced cerebral infarction in rats), acquired memory impairment models induced by D-galactose, scopolamine, reserpine, and alcohol, and platelets aggression model in rabbits. The development process of SLT is summarised in Figure 2.

Based on these studies, a new formulation was developed and standardized through a rigorous quality control program where 10 bioactive markers were quantified in the final product. The pharmacodynamic and pharmacokinetic 


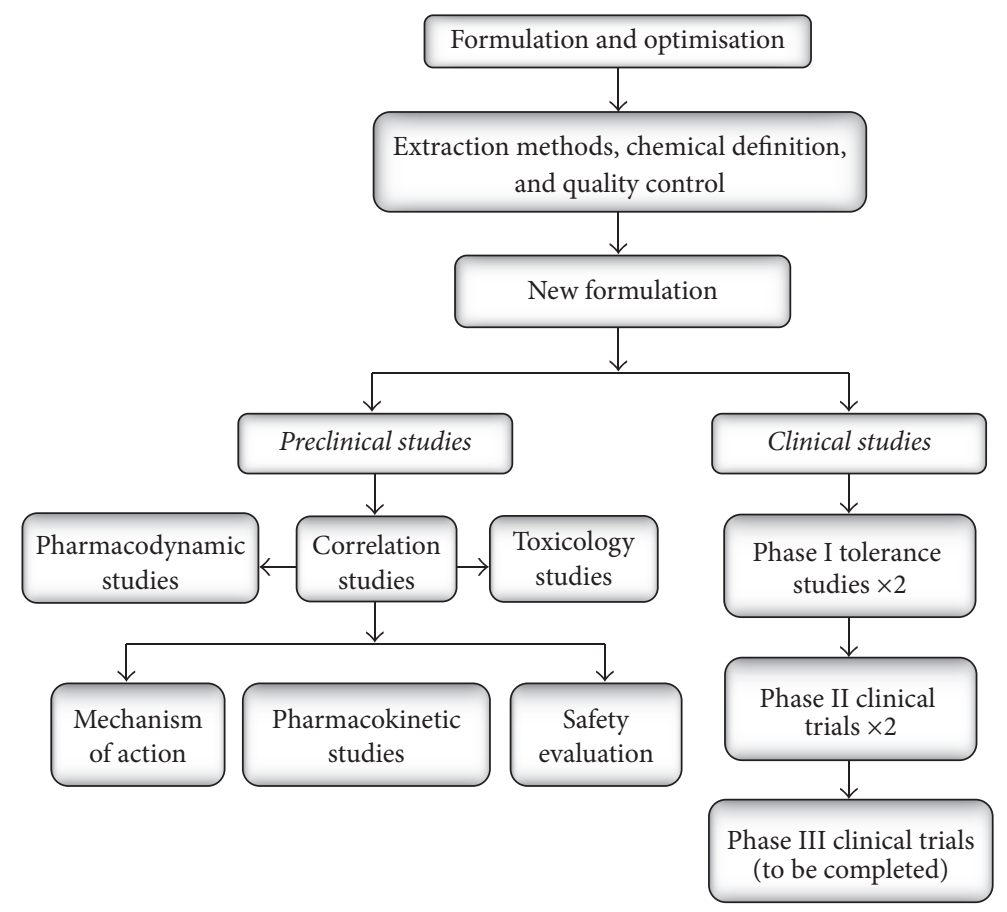

FIGURE 2: Development process of SLT, a novel, standardized complex herbal formulation for VaD.

properties of the formula were determined and the acute and chronic toxicity evaluated in animals. Human tolerability of the formula was determined in single administration and multiple (continuously) administration phase I studies and the clinical effectiveness, optimal clinical dose, and safety were determined in phase II studies. The efficacy and safety will be further evaluated in phase III studies where a greater number of $\mathrm{VaD}$ participants will be recruited.

5.2. Pharmacological Investigations of SLT. A series of preclinical pharmacokinetic and pharmacodynamic studies were conducted on SLT formula and its individual components. The data from these experiments demonstrated significant improvements in learning and memory function, pathogenic biochemical parameters in blood and brain tissue, and antioxidant capacity in various experimental dementia models.

In an in vivo study, the effect of SLT treatment (11, 22, and $44 \mathrm{mg} / \mathrm{kg}$ per day over 15 days) on memory impairment was investigated in acquired/consolidated dysmnesia models in mice induced by scopolamine, reserpine, chlorderazin, sodium nitrite, and alcohol. Compared with the control group, the medium and high dose treatments of SLT markedly decreased the error numbers and prolonged the latencies in all dysmnesia groups receiving active treatments, suggesting that SLT possesses beneficial protective effects on chemically induced cognitive impairments [98].

In a chronic cerebral hypoperfusion model induced by bilateral common carotid artery ligation in rats, 8-week treatment of SLT intragastrically (ig) significantly shortened the persistent time of finding the platform in a Morris water maze task [99]. Activity of cholinesterase was also significantly decreased $(p<0.05)$ while the acetylcholine (ACh) level was markedly increased in the brain tissue $(p<0.05)$. In addition, the activity of superoxide dismutase (SOD) was significantly enhanced $(p<0.05)$ [99]. These results suggest that SLT treatment improves hypoperfusioninduced cognitive impairments and this change may be associated with the cholinergic protective effect and free radical scavenging capacity of SLT formula.

The effects of SLT on ACh were also investigated in an amyloid $\beta$-protein induced dementia model in mice [52]. One-month treatment of SLT $(15.5 \mathrm{mg} / \mathrm{kg}$ and $31.0 \mathrm{mg} / \mathrm{kg}$ per day, ig) significantly increased the ACh levels in the brain tissue by $18.6 \%$ and $20.0 \%$, respectively, when compared with the model group. In another study, a longer treatment of SLT over 12 weeks at 31 and $62 \mathrm{mg} / \mathrm{kg}$ per day ig significantly increased the ACh levels in hippocampus in both treatment groups in a PDAPP ${ }^{\mathrm{v} 7171}$ transgenic dementia model in mice. In addition, hippocampal serotonin level was decreased significantly in the high dose SLT group [100].

The effects of crocin alone (the principal active component of Crocus sativus) on ischemia/reperfusion injury were investigated using a global or bilateral common carotid artery occlusion model in mice [101]. Pretreatment with $20 \mathrm{mg} / \mathrm{kg}$ crocin significantly inhibited oxidative stress (MDA content; $p<0.001$ ) in mice with $20 \mathrm{~min}$ of arterial occlusion followed by 24 hours of reperfusion. This change was accompanied by a significant elevation in total antioxidant capacity (increased SOD and glutathione peroxidase).

SLT has also demonstrated a range of cerebrovascular benefits. Acute SLT treatment over $24 \mathrm{~h}(8.25,16.5$, and 
$33 \mathrm{mg} / \mathrm{kg}$ ) decreased the areas of focal cerebral ischaemia/ reperfusion injury in rats and increased cerebral blood flow was also observed $60-180 \mathrm{~min}$ after administration $(10 \mathrm{mg} /$ $\mathrm{kg}$ ) [102]. Seven-day SLT treatment (16.5 and $8.25 \mathrm{mg} / \mathrm{kg}$ ) also showed a decrease in platelet aggregation rate and whole blood viscosity in rats [102].

In a pharmacokinetic study of SLT in rats, four bioactive components of ginkgo including bilobalide $\mathrm{B}$ and ginkgolides $\mathrm{A}, \mathrm{B}$, and $\mathrm{C}$ were found in rat plasma after oral ingestion $(60 \mathrm{mg} / \mathrm{kg})$, with a half-life between 1.6 and $2.8 \mathrm{~h}$ [101]. These constituents (especially bilobalide $\mathrm{B}$ and ginkgolide $\mathrm{A}$ ) were also found in brain tissue indicating that they were able to penetrate through the blood brain barrier into the brain tissue.

In an in vitro study, the antioxidant effect of SLT was investigated in cultured human vascular endothelial cell, EAhy926. SLT $(1-50 \mu \mathrm{g} / \mathrm{mL})$ significantly suppressed the $\mathrm{H}_{2} \mathrm{O}_{2}$-induced cell death and abolished the $\mathrm{H}_{2} \mathrm{O}_{2}$-induced ROS generation in a concentration dependent manner comparable to gallic acid $(10 \mu \mathrm{g} / \mathrm{mL})$, a well-known antioxidant [103]. In addition, SLT $(1-50 \mu \mathrm{g} / \mathrm{mL})$ significantly suppressed $\mathrm{H}_{2} \mathrm{O}_{2}$-induced $\mathrm{LDH}$ release. These results demonstrate that SLT has strong antioxidative and antiapoptotic activities on vascular endothelial cells. These properties may be associated with the neurological protective effects of SLT observed in people with $\mathrm{VaD}$.

5.3. Clinical Investigations of SLT. A phase 1 study of SLT was conducted in 54 participants for evaluation of tolerance and safety. In the single administration study $(60-540 \mathrm{mg} / \mathrm{dose}$, 30 participants), the following adverse events were observed: stomach discomfort, occurrence of urticaria, local skin pain, diarrhoea, itchy skin, dry mouth, heartburn, abdominal distension, dizziness, and nausea [104]. However, there was no significant difference in the proportion of these adverse events between the treatment group and the placebo group. Furthermore, the distribution of adverse events was not doserelated. In the continuous administration study, 24 healthy volunteers were randomised to receive a low $(180 \mathrm{mg} /$ day $)$ and a high dose (300 mg/day) treatment of SLT or equivalent doses of placebos over 14 days. There was no significant difference in the incidence rate of adverse events between the continuous treatment group and placebo group. No abnormal SLT treatment-related changes in liver and kidney function or ECG were observed. Overall, the results of the study showed that SLT was safe and well tolerated.

Using a randomised, double-blind, placebo-controlled crossover design, the effect of 1-week of SLT treatment on neurocognitive and cardiovascular function in 16 healthy adults was assessed [105]. In comparison to placebo, treatment with SLT resulted in a trend towards improvement in neuropsychological measures of working memory (immediate recall and N-back tasks) and in the brain's electrical response when attended information is encoded in memory (nonsignificant increase in P3a amplitude and significant decrease in N1 amplitude). The study showed that short-term SLT treatment is associated with more efficient attentional processing of auditory information and increased activation of working memory processes, suggesting that SLT has the potential to improve working memory performance in healthy adults.

In a phase II randomised, double-blinded, placebocontrolled pilot clinical study, 62 patients (32 in active group, 30 in placebo group) with probable or possible $\mathrm{VaD}$ were recruited according to the NINDS-AIREN criteria. Patients received 16-week treatment of either active compound or identical placebo after randomisation. At completion of the treatment, mean reductions in scores of the primary efficacy parameter, ADAS-cog, were $4.18 \pm 0.75$ and $1.18 \pm$ 0.58 in participants receiving SLT and placebo, respectively $[106,107]$. Although there is a difference in ADAS-cog at baseline (statistically nonsignificant) between the two groups, analysis of covariance (ANCOVA) showed that the improvement was significantly greater in SLT group than those in the placebo group after controlling for baseline ADAS-cog scores. A mechanistic substudy tested brain blood flow in 18 patients ( 7 patients SLT; 11 patients placebo) using Single Photon Emission Computed Tomography (SPECT). It was found that SLT treatment appeared to increase blood flow to the inferior frontal and anterior temporal lobes; regions associated with memory function and auditory and speech processing. This increase was more marked on the left when compared with the baseline in the treatment group only $[106,107]$. This study reported no serious adverse events.

A second phase II dose determination study of SLT (240 $\mathrm{mg}$ and $360 \mathrm{mg}$ per day over 52 weeks) in 325 patients with probable $\mathrm{VaD}$ has recently been completed. The preliminary analyses of the data indicate that 12-month treatment with SLT significantly improved cognitive function. No serious adverse reactions were reported. The full study report and publication are currently under preparation.

In summary, there are a range of challenges facing the development of complex herbal formulations. These challenges include (but are not limited to) selection of appropriate herbs, development of robust extraction methods and appropriate bioassay models, control of batch-tobatch quality, consistency of final products, establishment of pharmacokinetic and pharmacodynamic properties and toxicity profiles, and evaluation of effectiveness and safety through rigorous clinical trials. The SLT project provides an excellent example to demonstrate how some of these issues can be addressed. A considerable body of preclinical and clinical evidence has been constructed to support the use of SLT in VaD. Further work is now underway to further validate the effectiveness and safety of SLT with a greater $\mathrm{VaD}$ population in two multicentre phase III clinical trials. The preliminary trials detailed above provide promising findings that if supported by the phase III studies may lead to a breakthrough treatment for $\mathrm{VaD}$.

\section{Summary and Conclusion}

Preliminary evidence demonstrates the potential therapeutic benefits of herbal medicine either as single preparations or as complex herbal formulations for the treatment of dementia, $\mathrm{VaD}$, or mixed dementia. However, much of the evidence comes from animal and in vitro studies and overall clinical 
evidence to support these herbal interventions especially complex herbal preparations remains weak.

Multiple methodological issues have been identified in existing clinical trials. In addition to the general problems associated with sample size calculation, randomisation process, and statistical analysis of the results, several dementia/VaD trial specific issues also exist. For example, many trials did not specify the diagnostic criteria used to define AD, $\mathrm{VaD}$, and/or mixed dementia while, in other trials, diverse diagnostic criteria such as DSM-III, DSM-III-R, DSM-IV, ICD-10, ADDTC, HIS, and NINDS-AIREN were used. There is also a lack of consistency in the instruments used for assessment of cognitive function and some instruments that were used have not been validated in dementia and/or VaD cohorts.

The treatment durations in the existing trials are also of concern. Dementia is a progressive disease and therefore longer duration clinical trials are required to appropriately assess the effects of interventions on the disease progress. The guidelines on medical products for $\mathrm{AD}$ and other dementias published by the European Medicines Agency mandate that controlled clinical trials aiming at demonstrating short-term improvement in $\mathrm{AD}$ and $\mathrm{VaD}$ should last at least 6 months and 12 months, respectively [108]. In addition to cognitive functions, the changes in ADL, global clinical improvement, quality of life, neuropsychiatric symptoms, and carer burden should also ideally be investigated in the future trials.

Few mechanistic studies exist to assess the synergistic effects among the multiple herbs and/or multiple active components. This is by and large caused by the lack of robust methods to evaluate synergy. As such, more research is urgently needed in this area. Herbal medicine, particularly Chinese herbal medicine, has been widely used for the control of various risk factors such as hypertension, atherosclerosis, and diabetes associated with cardiovascular disease and $\mathrm{VaD}$. However, no studies were identified that assessed the prevention of $\mathrm{VaD}$ using herbal medicines. A number of studies evaluating the conversion of mild cognitive impairment to $\mathrm{AD}$ using long-term ginkgo treatment failed to demonstrate positive outcomes $[109,110]$. Future epidemiological and clinical studies are required to further assess the benefits of herbal medicines for the prevention of dementia and/or $\mathrm{VaD}$.

In conclusion, the existing evidence to support the use of single and complex herbal preparations is promising but requires further development. There are numerous issues relating to the trial design in clinical studies of herbal medicines for dementia and VaD. The case study outlined here demonstrates the feasibility and potential of developing evidence-based herbal medicines for the treatment of VaD.

\section{Competing Interests}

The authors have received funding support from universities, government agencies, and industry in China and Australia in the development of SLT formula (as discussed in Section 5). The funding bodies include Ministry of Science and Technology of China, National Natural Science Foundation of China, Academy of Chinese Medical Sciences of
China, Shineway Pharmaceutical Group, National Health and Medical Research Council of Australia, and Western Sydney University.

\section{Authors' Contributions}

Dennis Chang and Jianxun Liu contributed equally to this work. All other authors have made significant contributions in the preparation of this manuscript.

\section{References}

[1] Dementia Fact Sheet, World Health Organisation, 2016.

[2] "Australian Institute of Health and Welfare 2012," Dementia in Australia. Cat. no. AGE 70. Canberra, Canada: AIHW, 2012.

[3] Australian Bureau of Statistics, 3303.0-Causes of Death, Australia, Australian Bureau of Statistics, Canberra, Australia, 2014.

[4] R. N. Kalaria, G. E. Maestre, R. Arizaga et al., "Alzheimer's disease and vascular dementia in developing countries: prevalence, management, and risk factors," The Lancet Neurology, vol. 7, no. 9, pp. 812-826, 2008.

[5] K. A. Nolan, M. M. Lino, A. W. Seligmann, and J. P. Blass, "Absence of vascular dementia in an autopsy series from a dementia clinic," Journal of the American Geriatrics Society, vol. 46, no. 5, pp. 597-604, 1998.

[6] M. R. Farlow, M. L. Miller, and V. Pejovic, "Treatment options in Alzheimer's disease: maximizing benefit, managing expectations," Dementia and Geriatric Cognitive Disorders, vol. 25, no. 5, pp. 408-422, 2008.

[7] J. Liu and D. Chang, "Vascular dementia," Journal of Complementary Medicine, vol. 5, pp. 14-20, 2006.

[8] D. A. Levine and K. M. Langa, "Vascular cognitive impairment: disease mechanisms and therapeutic implications," Neurotherapeutics, vol. 8, no. 3, pp. 361-373, 2011.

[9] I. Skoog, "Status of risk factors for vascular dementia," Neuroepidemiology, vol. 17, no. 1, pp. 2-9, 1998.

[10] N. T. Aggarwal and C. DeCarli, "Vascular dementia: emerging trends," Seminars in Neurology, vol. 27, no. 1, pp. 66-77, 2007.

[11] P. Sachdev and H. Brodaty, "Vascular dementia: an Australian perspective," Alzheimer Disease and Associated Disorders, vol. 13, supplement 3, pp. S206-S212, 1999.

[12] H. Shim, "Vascular cognitive impairment and post-stroke cognitive deficits," Current Neurology and Neuroscience Reports, vol. 14, no. 1, article 418, 2014.

[13] J. V. Smith and Y. Luo, "Studies on molecular mechanisms of Ginkgo biloba extract," Applied Microbiology and Biotechnology, vol. 64, no. 4, pp. 465-472, 2004.

[14] P. C. Chan, Q. Xia, and P. P. Fu, "Ginkgo biloba leave extract: biological, medicinal, and toxicological effects," Journal of Environmental Science and Health. Part C, Environmental Carcinogenesis \& Ecotoxicology Reviews, vol. 25, no. 3, pp. 211244, 2007.

[15] J. Wang, W. Chen, and Y. Wang, "A ginkgo biloba extract promotes proliferation of endogenous neural stem cells in vascular dementia rats," Neural Regeneration Research, vol. 8, no. 18, pp. 1655-1662, 2013.

[16] L.-Y. Zhang and Y.-L. Wang, "[Effects of EGb761 on hippocamal synaptic plasticity of vascular dementia rats]," Chinese journal of applied physiology, vol. 24, no. 1, pp. 36-40, 2008. 
[17] Z.-X. Yao, Z. Han, K. Drieu, and V. Papadopoulos, "Ginkgo biloba extract (Egb 761) inhibits $\beta$-amyloid production by lowering free cholesterol levels," Journal of Nutritional Biochemistry, vol. 15, no. 12, pp. 749-756, 2004.

[18] M. Hrehorovská, J. Burda, I. Domoráková, and E. Mechírová, "Effect of Tanakan on postischemic activity of protein synthesis machinery in the rat brain," General Physiology and Biophysics, vol. 23, no. 4, pp. 457-465, 2004.

[19] P.-O. Koh, "Gingko biloba extract (EGb 761) prevents cerebral ischemia-induced p70S6 kinase and S6 phosphorylation," American Journal of Chinese Medicine, vol. 38, no. 4, pp. 727734, 2010.

[20] S. Saleem, H. Zhuang, S. Biswal, Y. Christen, and S. Doré, "Ginkgo biloba extract neuroprotective action is dependent on heme oxygenase 1 in ischemic reperfusion brain injury," Stroke, vol. 39, no. 12, pp. 3389-3396, 2008.

[21] B. Spinnewyn, N. Blavet, and F. Clostre, "[Effects of ginkgo biloba extract on a cerebral ischemia model in gerbils]," Presse Medicale, vol. 15, no. 31, pp. 1511-1515, 1986.

[22] M.-N. Rocher, D. Carré, B. Spinnewyn et al., "Long-term treatment with standardized Ginkgo biloba Extract (EGb 761) attenuates cognitive deficits and hippocampal neuron loss in a gerbil model of vascular dementia," Fitoterapia, vol. 82, no. 7, pp. 1075-1080, 2011.

[23] W.-Z. Li, W.-Y. Wu, H. Huang, Y.-Y. Wu, and Y.-Y. Yin, "Protective effect of bilobalide on learning and memory impairment in rats with vascular dementia," Molecular Medicine Reports, vol. 8, no. 3, pp. 935-941, 2013.

[24] A. B. Scholey and D. O. Kennedy, "Acute, dose-dependent cognitive effects of Ginkgo biloba, Panax ginseng and their combination in healthy young volunteers: differential interactions with cognitive demand," Human Psychopharmacology, vol. 17, no. 1, pp. 35-44, 2002.

[25] S.-J. Zhang and Z.-Y. Xue, "Effect of Western medicine therapy assisted by Ginkgo biloba tablet on vascular cognitive impairment of none dementia," Asian Pacific Journal of Tropical Medicine, vol. 5, no. 8, pp. 661-664, 2012.

[26] L. S. Schneider, "Ginkgo biloba extract and preventing Alzheimer disease," JAMA, vol. 300, no. 19, pp. 2306-2308, 2008.

[27] S. Kanowski, W. M. Herrmann, K. Stephan, W. Wierich, and R. Hörr, "Proof of efficacy of the ginkgo biloba special extract EGb 761 in outpatients suffering from mild to moderate primary degenerative dementia of the Alzheimer type or multi-infarct dementia," Pharmacopsychiatry, vol. 29, no. 2, pp. 47-56, 1996.

[28] R. Ihl, M. Tribanek, N. Bachinskaya, and Gotaday Study Group, "Efficacy and tolerability of a once daily formulation of Ginkgo biloba extract EGb 761(R) in Alzheimer's disease and vascular dementia: results from a randomised controlled trial," Pharmacopsychiatry, vol. 45, no. 2, pp. 41-46, 2012.

[29] S. Gauthier and S. Schlaefke, "Efficacy and tolerability of Ginkgo biloba extract EGb $761^{\circledR}$ in dementia: a systematic review and meta-analysis of randomized placebo-controlled trials," Clinical Interventions in Aging, vol. 9, pp. 2065-2077, 2014.

[30] M.-S. Tan, J.-T. Yu, C.-C. Tan et al., "Efficacy and adverse effects of Ginkgo Biloba for cognitive impairment and dementia: a systematic review and meta-analysis," Journal of Alzheimer's Disease, vol. 43, no. 2, pp. 589-603, 2015.

[31] Y. Wang, L.-Q. Huang, X.-C. Tang, and H.-Y. Zhang, "Retrospect and prospect of active principles from Chinese herbs in the treatment of dementia," Acta Pharmacologica Sinica, vol. 31, no. 6, pp. 649-664, 2010.
[32] M.-J. R. Howes and P. J. Houghton, "Plants used in Chinese and Indian traditional medicine for improvement of memory and cognitive function," Pharmacology Biochemistry and Behavior, vol. 75, no. 3, pp. 513-527, 2003.

[33] H. Y. Zhang, C. Y. Zheng, H. Yan et al., "Potential therapeutic targets of huperzine A for Alzheimer's disease and vascular dementia," Chemico-Biological Interactions, vol. 175, no. 1-3, pp. 396-402, 2008.

[34] B.-S. Wang, H. Wang, Z.-H. Wei, Y.-Y. Song, L. Zhang, and H.-Z. Chen, "Efficacy and safety of natural acetylcholinesterase inhibitor huperzine A in the treatment of Alzheimer's disease: an updated meta-analysis," Journal of Neural Transmission, vol. 116, no. 4, pp. 457-465, 2009.

[35] S. H. Adams, C. L. Hoppel, K. H. Lok et al., "Plasma acylcarnitine profiles suggest incomplete long-chain fatty acid $\beta$ oxidation and altered tricarboxylic acid cycle activity in type 2 diabetic African-American women," Journal of Nutrition, vol. 139, no. 6, pp. 1073-1081, 2009.

[36] G. Yang, Y. Wang, J. Tian, and J.-P. Liu, "Huperzine A for Alzheimer's disease: a systematic review and meta-analysis of randomized clinical trials," PLoS ONE, vol. 8, no. 9, Article ID e74916, 2013.

[37] Z. Hao, M. Liu, Z. Liu, and D. Lv, "Huperzine A for vascular dementia," Cochrane Database of Systematic Reviews, no. 2, Article ID CD007365, 2009.

[38] S. H. Xing, C.-X. Zhu, R. Zhang, and L. An, "Huperzine a in the treatment of Alzheimer's disease and vascular dementia: a meta-analysis," Evidence-Based Complementary and Alternative Medicine, vol. 2014, Article ID 363985, 10 pages, 2014.

[39] A. Goel, A. B. Kunnumakkara, and B. B. Aggarwal, "Curcumin as 'Curecumin': from kitchen to clinic," Biochemical Pharmacology, vol. 75, no. 4, pp. 787-809, 2008.

[40] J. M. Ringman, S. A. Frautschy, G. M. Cole, D. L. Masterman, and J. L. Cummings, "A potential role of the curry spice curcumin in Alzheimer's disease," Current Alzheimer Research, vol. 2, no. 2, pp. 131-136, 2005.

[41] F. Yang, G. P. Lim, A. N. Begum et al., "Curcumin inhibits formation of amyloid $\beta$ oligomers and fibrils, binds plaques, and reduces amyloid in vivo," The Journal of Biological Chemistry, vol. 280, no. 7, pp. 5892-5901, 2005.

[42] T.-P. Ng, P.-C. Chiam, T. Lee, H.-C. Chua, L. Lim, and E.-H. Kua, "Curry consumption and cognitive function in the elderly," American Journal of Epidemiology, vol. 164, no. 9, pp. 898-906, 2006.

[43] H. Hatcher, R. Planalp, J. Cho, F. M. Torti, and S. V. Torti, "Curcumin: from ancient medicine to current clinical trials," Cellular and Molecular Life Sciences, vol. 65, no. 11, pp. 1631-1652, 2008.

[44] L. Baum, C. W. K. Lam, S. K.-K. Cheung et al., "Six-month randomized, placebo-controlled, double-blind, pilot clinical trial of curcumin in patients with Alzheimer disease [7]," Journal of Clinical Psychopharmacology, vol. 28, no. 1, pp. 110113, 2008.

[45] J. M. Ringman, G. Cole, E. Teng et al., "Oral curcumin for the treatment of mild-to-moderate Alzheimer's disease: tolerability and clinical and biomarker efficacy results of a placebocontrolled 24-week study," in Proceedings of the Abstract of International Conference on Alzheimer's Disease, Chicago, Ill, USA, 2008. 
[46] J. M. Ringman, S. A. Frautschy, E. Teng et al., "Oral curcumin for Alzheimer's disease: tolerability and efficacy in a 24-week randomized, double blind, placebo-controlled study," Alzheimer's Research and Therapy, vol. 4, no. 5, article 43, 2012.

[47] C.-M. Chuang, C.-L. Hsieh, H.-Y. Lin, and J.-G. Lin, "Panax Notoginseng Burk attenuates impairment of learning and memory functions and increases ED1, BDNF and $\beta$-secretase immunoreactive cells in chronic stage ischemia-reperfusion injured rats," American Journal of Chinese Medicine, vol. 36, no. 4, pp. 685-693, 2008.

[48] R. C. Y. Choi, J. T. T. Zhu, K. W. Leung et al., "A flavonol glycoside, isolated from roots of panax notoginseng, reduces amyloid- $\beta$-induced neurotoxicity in cultured neurons: signaling transduction and drug development for Alzheimer's disease," Journal of Alzheimer's Disease, vol. 19, no. 3, pp. 795811, 2010.

[49] K. Radad, G. Gille, L. Liu, and W.-D. Rausch, "Use of ginseng in medicine with emphasis on neurodegenerative disorders," Journal of Pharmacological Sciences, vol. 100, no. 3, pp. 175-186, 2006.

[50] S. Chu, J. Gu, L. Feng et al., "Ginsenoside Rg5 improves cognitive dysfunction and beta-amyloid deposition in STZinduced memory impaired rats via attenuating neuroinflammatory responses," International Immunopharmacology, vol. 19, no. 2, pp. 317-326, 2014.

[51] H. Yang, J. Zhang, R. M. Breyer, and C. Chen, "Altered hippocampal long-term synaptic plasticity in mice deficient in the PGE2 EP2 receptor," Journal of Neurochemistry, vol. 108, no. 1, pp. 295-304, 2009.

[52] J.-X. Liu, W.-H. Cong, L. Xu, and J.-N. Wang, "Effect of combination of extracts of ginseng and ginkgo biloba on acetylcholine in amyloid beta-protein-treated rats determined by an improved HPLC," Acta Pharmacologica Sinica, vol. 25, no. 9, pp. 1118-1123, 2004.

[53] J. Shi, S. Zhang, M. Tang et al., “The 1239G/C polymorphism in exon 5 of BACE1 gene may be associated with sporadic Alzheimer's disease in Chinese Hans," American Journal of Medical Genetics Part B: Neuropsychiatric Genetics, vol. 124, no. 1, pp. 54-57, 2004.

[54] Y. Sun, J. Ke, N. Ma, Z. Chen, C. Wang, and X. Cui, "[Effects of root rot on saponin content in Panax notoginseng]," Zhong yao cai $=$ Zhongyaocai $=$ Journal of Chinese medicinal materials, vol. 27, no. 2, pp. 79-80, 2004.

[55] K.-T. Choi, "Botanical characteristics, pharmacological effects and medicinal components of Korean Panax ginseng C A Meyer," Acta Pharmacologica Sinica, vol. 29, no. 9, pp. 1109-1118, 2008.

[56] J. L. Reay, D. O. Kennedy, and A. B. Scholey, "Effects of Panax ginseng, consumed with and without glucose, on blood glucose levels and cognitive performance during sustained 'mentally demanding' tasks," Journal of Psychopharmacology, vol. 20, no. 6, pp. 771-781, 2006.

[57] D. O. Kennedy, A. B. Scholey, L. Drewery, V. R. Marsh, B. Moore, and H. Ashton, "Electroencephalograph effects of single doses of Ginkgo biloba and Panax ginseng in healthy young volunteers," Pharmacology Biochemistry and Behavior, vol. 75, no. 3, pp. 701-709, 2003.

[58] J.-H. Heo, S.-T. Lee, K. Chu et al., "An open-label trial of Korean red ginseng as an adjuvant treatment for cognitive impairment in patients with Alzheimer's disease," European Journal of Neurology, vol. 15, no. 8, pp. 865-868, 2008.
[59] S.-T. Lee, K. Chu, J.-Y. Sim, J.-H. Heo, and M. Kim, "Panax ginseng enhances cognitive performance in Alzheimer disease," Alzheimer Disease and Associated Disorders, vol. 22, no. 3, pp. 222-226, 2008.

[60] J.-H. Heo, S.-T. Lee, M. J. Oh et al., "Improvement of cognitive deficit in Alzheimer's disease patients by long term treatment with Korean red ginseng," Journal of Ginseng Research, vol. 35, no. 4, pp. 457-461, 2011.

[61] G. Z. Steiner, A. Yeung, J.-X. Liu et al., "The effect of sailuotong (SLT) on neurocognitive and cardiovascular function in healthy adults: a randomised, double-blind, placebo controlled crossover pilot trial," BMC Complementary and Alternative Medicine, vol. 16, article 15, 2016.

[62] K. A. Wesnes, T. Ward, A. McGinty, and O. Petrini, "The memory enhancing effects of a Ginkgo biloba/Panax ginseng combination in healthy middle-aged volunteers," Psychopharmacology, vol. 152, no. 4, pp. 353-361, 2000.

[63] D. O. Kennedy, A. B. Scholey, and K. A. Wesnes, "Differential, dose dependent changes in cognitive performance following acute administration of a Ginkgo biloba/Panax ginseng combination to healthy young volunteers," Nutritional Neuroscience, vol. 4, no. 5, pp. 399-412, 2001.

[64] J. Tian, "Ginseng may improve memory in stroke dementia patients," in Proceedings of the American Stroke Association Meeting, Augusta, Canada, 2003.

[65] Q. F. Gui, Y. M. Yang, S. H. Ying, and M. M. Zhang, "Xueshuantong improves cerebral blood perfusion in elderly patients with lacunar infarction," Neural Regeneration Research, vol. 8, no. 9, pp. 792-801, 2013.

[66] A. Russo and F. Borrelli, "Bacopa monniera, a reputed nootropic plant: an overview," Phytomedicine, vol. 12, no. 4, pp. 305-317, 2005.

[67] S. K. Bhattacharya, A. Bhattacharya, A. Kumar, and S. Ghosal, "Antioxidant activity of Bacopa monniera in rat frontal cortex, striatum and hippocampus," Phytotherapy Research, vol. 14, no. 3, pp. 174-179, 2000.

[68] A. Russo, A. A. Izzo, F. Borrelli, M. Renis, and A. Vanella, "Free radical scavenging capacity and protective effect of Bacopa monniera L. on DNA damage," Phytotherapy Research, vol. 17, no. 8, pp. 870-875, 2003.

[69] Y. B. Tripathi, S. Chaurasia, E. Tripathi, A. Upadhyay, and G. P. Dubey, "Bacopa monniera Linn. as an antioxidant: mechanism of action," Indian Journal of Experimental Biology, vol. 34, no. 6, pp. 523-526, 1996.

[70] N. Kamkaew, C. N. Scholfield, K. Ingkaninan, N. Taepavarapruk, and K. Chootip, "Bacopa monnieri increases cerebral blood flow in rat independent of blood pressure," Phytotherapy Research, vol. 27, no. 1, pp. 135-138, 2013.

[71] D. Kar Chowdhuri, D. Parmar, P. Kakkar, R. Shukla, P. K. Seth, and R. C. Srimal, "Antistress effects of bacosides of Bacopa monnieri: modulation of Hsp70 expression, superoxide dismutase and cytochrome $\mathrm{P} 450$ activity in rat brain," Phytotherapy Research, vol. 16, no. 7, pp. 639-645, 2002.

[72] K. Kishore and M. Singh, "Effect of bacosides, alcoholic extract of Bacopa monniera Linn. (brahmi), on experimental amnesia in mice," Indian Journal of Experimental Biology, vol. 43, no. 7, pp. 640-645, 2005.

[73] H. K. Singh and B. N. Dhawan, "Neuropsychopharmacological effects of the Ayurvedic nootropic Bacopa monniera linn (Brahmi)," Indian Journal of Pharmacology, vol. 29, no. 5, pp. 359-365, 1997. 
[74] C. Stough, A. Scholey, V. Cropley et al., "Examining the cognitive effects of a special extract of Bacopa monniera (CDRI08: Keenmind): a review of ten years of research at Swinburne University," Journal of Pharmacy and Pharmaceutical Sciences, vol. 16, no. 2, pp. 254-258, 2013.

[75] M. P. Pase, J. Kean, J. Sarris, C. Neale, A. B. Scholey, and C. Stough, "The cognitive-enhancing effects of bacopa monnieri: a systematic review of randomized, controlled human clinical trials," Journal of Alternative and Complementary Medicine, vol. 18, no. 7, pp. 647-652, 2012.

[76] C. K. Stough, M. P. Pase, V. Cropley et al., "A randomized controlled trial investigating the effect of Pycnogenol and Bacopa CDRI08 herbal medicines on cognitive, cardiovascular, and biochemical functioning in cognitively healthy elderly people: the Australian Research Council Longevity Intervention (ARCLI) study protocol (ANZCTR12611000487910)," Nutrition Journal, vol. 11, article 11, 2012.

[77] B. Amin, K. Abnous, V. Motamedshariaty, and H. Hosseinzadeh, "Attenuation of oxidative stress, inflammation and apoptosis by ethanolic and aqueous extracts of Crocus sativus $\mathrm{L}$. stigma after chronic constriction injury of rats," Anais da Academia Brasileira de Ciências, vol. 86, no. 4, pp. 1821-1832, 2014.

[78] K. Abe and H. Saito, "Effects of saffron extract and its constituent crocin on learning behaviour and long-term potentiation," Phytotherapy Research, vol. 14, no. 3, pp. 149-152, 2000.

[79] H. Hosseinzadeh and T. Ziaei, "Effects of Crocus sativus stigma extract and its constituents, crocin and safranal, on intact memory and scopolamine-induced learning deficits in rats performing the Morris water maze task," Journal of Medicinal Plants, vol. 5, no. 19, pp. 40-50, 2006.

[80] H. Hosseinzadeh and H. R. Sadeghnia, "Safranal, a constituent of Crocus sativus (saffron), attenuated cerebral ischemia induced oxidative damage in rat hippocampus," Journal of Pharmacy and Pharmaceutical Sciences, vol. 8, no. 3, pp. 394399, 2005.

[81] S. W. Jessie and T. P. Krishnakantha, "Inhibition of human platelet aggregation and membrane lipid peroxidation by food spice, saffron," Molecular and Cellular Biochemistry, vol. 278, no. 1, pp. 59-63, 2005.

[82] “Ancient Chinese pharmacopoeia," Il Policlinico. Sezione Pratica, vol. 57, no. 34, pp. 1108-1109, 1950.

[83] S. Akhondzadeh, M. S. Sabet, M. H. Harirchian et al., "A 22-week, multicenter, randomized, double-blind controlled trial of Crocus sativus in the treatment of mild-to-moderate Alzheimer's disease," Psychopharmacology, vol. 207, no. 4, pp. 637-643, 2010.

[84] S. Akhondzadeh, M. S. Sabet, M. H. Harirchian et al., "Saffron in the treatment of patients with mild to moderate Alzheimer's disease: a 16-week, randomized and placebo-controlled trial," Journal of Clinical Pharmacy and Therapeutics, vol. 35, no. 5, pp. 581-588, 2010.

[85] S. A. Mandel, T. Amit, L. Kalfon, L. Reznichenko, and M. B. H. Youdim, "Targeting multiple neurodegenerative diseases etiologies with multimodal-acting green tea catechins," Journal of Nutrition, vol. 138, no. 8, pp. 1578S-1583S, 2008.

[86] A. B. Sharangi, "Medicinal and therapeutic potentialities of tea (Camellia sinensis L.) - a review," Food Research International, vol. 42, no. 5-6, pp. 529-535, 2009.

[87] S. Kuriyama, A. Hozawa, K. Ohmori et al., "Green tea consumption and cognitive function: a cross-sectional study from the
Tsurugaya Project 1," American Journal of Clinical Nutrition, vol. 83, no. 2, pp. 355-361, 2006.

[88] A. Schmidt, F. Hammann, B. Wölnerhanssen et al., "Green tea extract enhances parieto-frontal connectivity during working memory processing," Psychopharmacology, vol. 231, no. 19, pp. 3879-3888, 2014.

[89] M. Noguchi-Shinohara, S. Yuki, C. Dohmoto et al., "Consumption of green tea, but not black tea or coffee, is associated with reduced risk of cognitive decline," PLOS ONE, vol. 9, no. 5, Article ID e96013, 2014.

[90] K. Ide, H. Yamada, N. Takuma et al., "Effects of green tea consumption on cognitive dysfunction in an elderly population: a randomized placebo-controlled study," Nutrition Journal, vol. 15, no. 1, article 49, 2016.

[91] H. Wagner and G. Ulrich-Merzenich, "Synergy research: approaching a new generation of phytopharmaceuticals," Phytomedicine, vol. 16, no. 2-3, pp. 97-110, 2009.

[92] X. Zhou, S. W. Seto, D. Chang et al., "Synergistic effects of chinese herbal medicine: a comprehensive review of methodology and current research," Frontiers in Pharmacology, vol. 7, article 201, 2016.

[93] P. Gu and H. Chen, "Modern bioinformatics meets traditional Chinese medicine," Briefings in Bioinformatics, vol. 15, no. 6, Article ID bbt063, pp. 984-1003, 2013.

[94] K. Iwasaki, S. Kobayashi, Y. Chimura et al., "A randomized, double-blind, placebo-controlled clinical trial of the Chinese herbal medicine 'Ba Wei Di Huang Wan' in the treatment of dementia," Journal of the American Geriatrics Society, vol. 52, no. 9, pp. 1518-1521, 2004.

[95] K. Nagata, E. Yokoyama, T. Yamazaki et al., "Effects of yokukansan on behavioral and psychological symptoms of vascular dementia: an open-label trial," Phytomedicine, vol. 19, no. 6, pp. 524-528, 2012.

[96] S. C. Man, K. W. Chan, J. Lu, S. S. Durairajan, L. Liu, and M. Li, "Systematic review on the efficacy and safety of herbal medicines for vascular dementia," Evidence-Based Complementary and Alternative Medicine, vol. 2012, Article ID 426215, 22 pages, 2012.

[97] D. Gong, J. Xu, and Y. Fan, "Meta-analysis of clinical trials of oral Chinese herbal prescriptions for treatment of vascular dementia based on mini mental state examination scores," European Journal of Integrative Medicine, vol. 7, no. 2, pp. 108117, 2015.

[98] L. Xu, W. Cong, C. Wei, and J. Liu, "Effects of Weinaokang (SLT) on dysmnesia in mice models," Pharmacology and Clinics of Chinese Materia Medica, no. 6, pp. 60-62, 2007.

[99] L. Xu, J.-X. Liu, W.-H. Cong, and C.-E. Wei, "[Effects of Weinaokang capsule on intracephalic cholinergic system and capability of scavenging free radicas in chronic cerebral hypoperfusion rats]," Zhongguo Zhongyao Zazhi, vol. 33, no. 5, pp. 531534, 2008.

[100] W. H. Cong, J. X. Liu, and L. Xu, "Effects of extracts of Ginseng and Ginkgo biloba on hippocampal acetylcholine and monoamines in PDAP-pV717I transgenic mice," Zhongguo Zhong xi yi jie he za zhi Zhongguo Zhongxiyi jiehe zazhi, vol. 27, no. 9, pp. 810-813, 2007.

[101] Y. Zhang, L. Lin, C. Ren et al., "Pharmacokinetics and brain distribution of ginkgolides after administration of Sai-LuoTong," Modernization of Traditional Chinese Medicine and Materia Medica, vol. 16, no. 7, pp. 1458-1464, 2014. 
[102] W.-H. Cong, B. Yang, L. Xu et al., "Herbal extracts combination (WNK) prevents decline in spatial learning and memory in APP/PS1 mice through improvement of hippocampal A $\beta$ plaque formation, histopathology, and ultrastructure," Evidence-based Complementary and Alternative Medicine, vol. 2012, Article ID 478190, 9 pages, 2012.

[103] S. Seto, A. Jenkins, H. Kiat, A. Bensoussan, J. Liu, and D. Chang, "Protective effects of a standardised herbal formulation, Sailotong, on hydrogen peroxide $\left(\mathrm{H}_{2} \mathrm{O}_{2}\right)$ induced damage in EA.HY926 Cells," The Journal of Alternative and Complementary Medicine, vol. 22, no. 6, p. A35, 2016.

[104] T. Li, H.-M. Liu, Y. Lu et al., "A phase I tolerance and safety study of Sailuotong capsule," Chinese Journal of New Drugs, vol. 21, no. 1, pp. 62-67, 2012.

[105] G. Z. Steiner, A. Yeung, J.-X. Liu et al., "The effect of Sailuotong (SLT) on neurocognitive and cardiovascular function in healthy adults: a randomised, double-blind, placebo controlled crossover pilot trial," BMC Complementary and Alternative Medicine, vol. 16, no. 15, 2016.

[106] J. Liu, D. Chang, D. Chan, J. Liu, and A. Bensoussan, "A randomised placebo-controlled clinical trial of a Chinese herbal medicine for the treatment of vascular dementia," in Proceedings of the 2nd International Congress for Complementary Medicine Research, Munich, Germany, 2007.

[107] D. Chang, B. Colagiuri, and R. Luo, Chinese Medicine used to Treat Dementia, Advances in Natural Medicines, Nutraceuticals and Neurocognition, CRC Press, 2013.

[108] European Medicines Agency, Guideline on Medicinal Products for the Treatment of Alzheimer's Disease and other Dementias, European Medicines Agency, London, UK, 2008.

[109] B. Vellas, N. Coley, P.-J. Ousset et al., "Long-term use of standardised Ginkgo biloba extract for the prevention of Alzheimer's disease (GuidAge): a randomised placebocontrolled trial," The Lancet Neurology, vol. 11, no. 10, pp. 851859, 2012.

[110] B. E. Snitz, E. S. O’Meara, M. C. Carlson et al., "Ginkgo biloba for preventing cognitive decline in older adults: a randomized trial," JAMA-Journal of the American Medical Association, vol. 302, no. 24, pp. 2663-2670, 2009. 


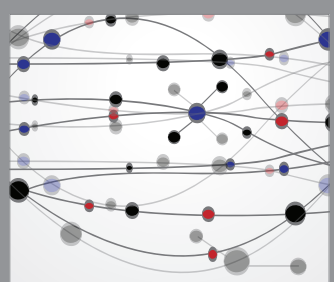

The Scientific World Journal
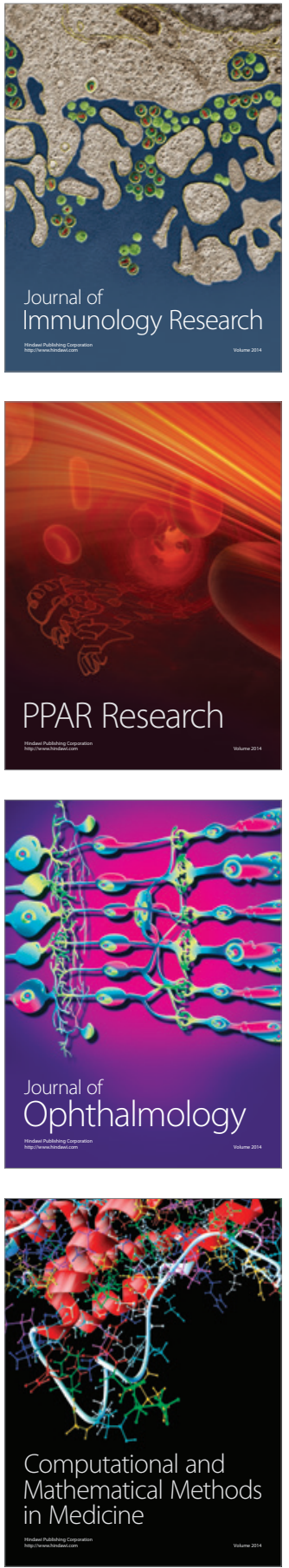

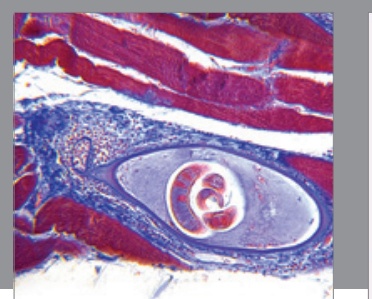

Gastroenterology Research and Practice

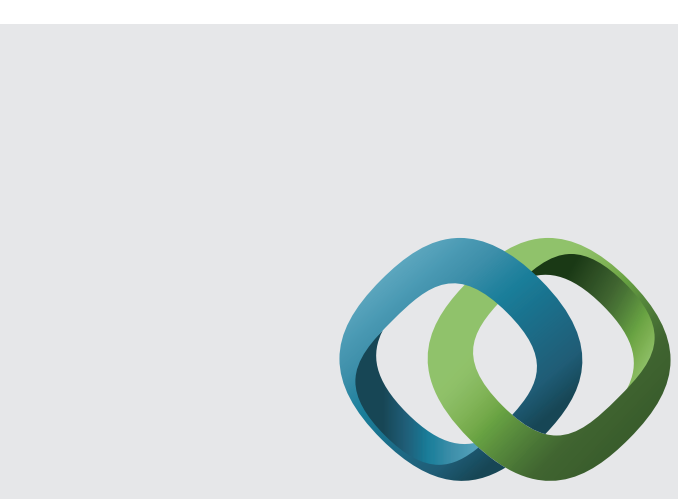

\section{Hindawi}

Submit your manuscripts at

http://www.hindawi.com
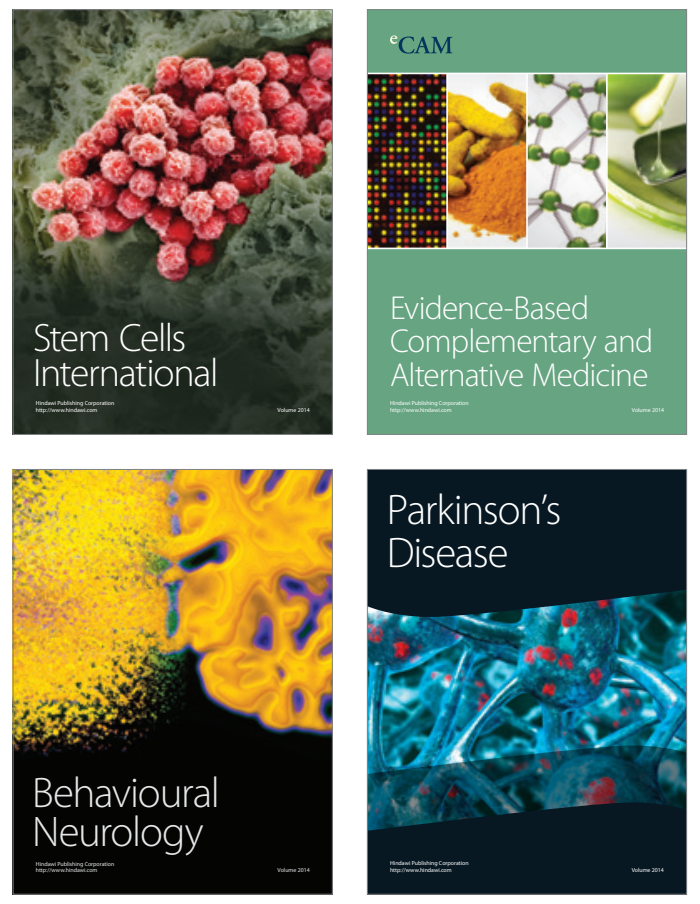
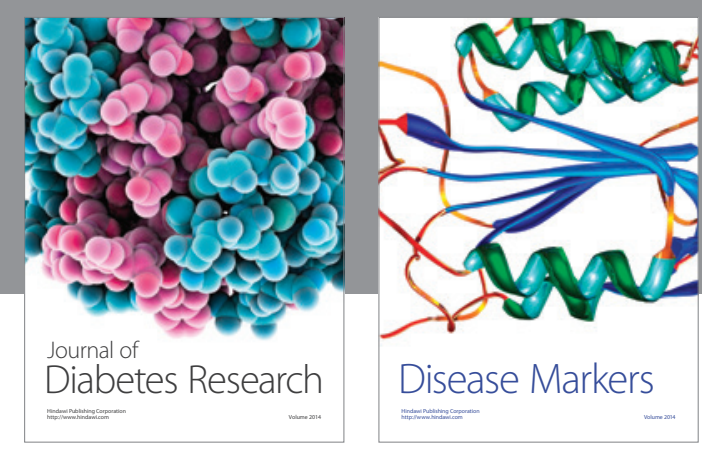

Disease Markers
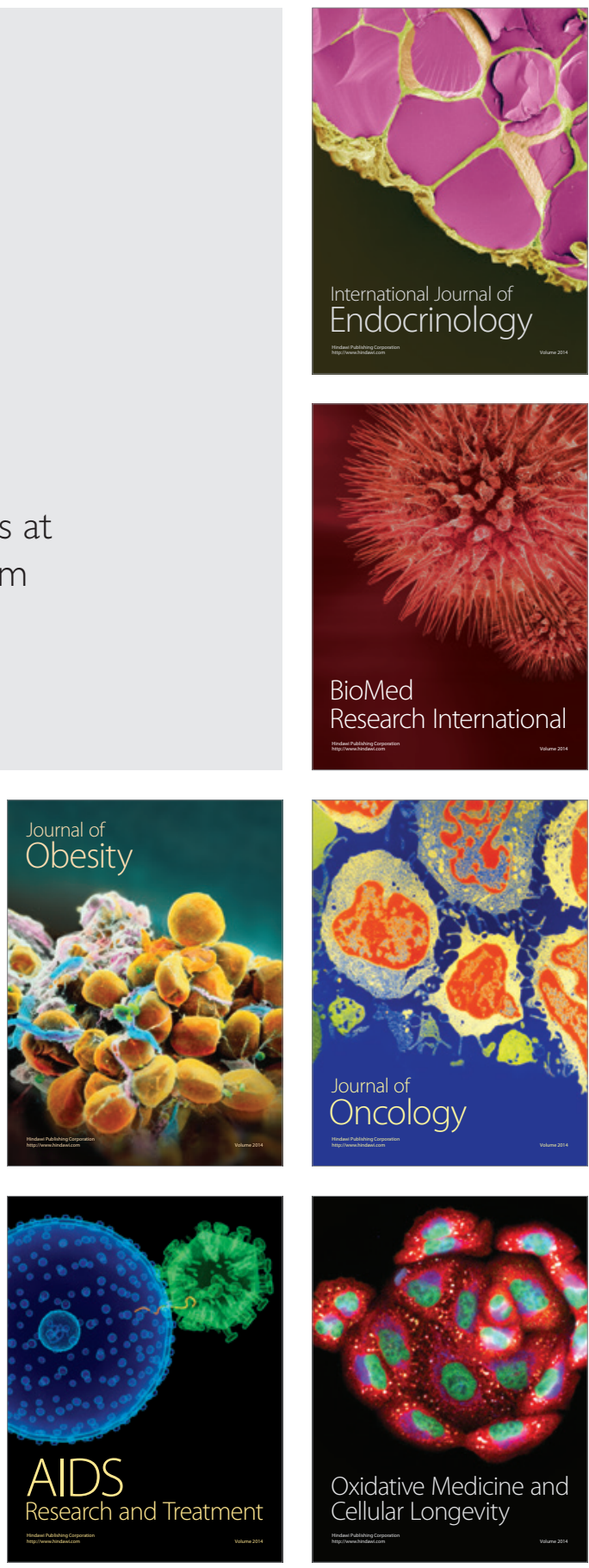